\title{
Phases of the disordered Bose-Hubbard model with attractive interactions
}

\author{
Olli Mansikkamäki ๑, Sami Laine $\odot$, and Matti Silveri $\odot$ \\ Nano and Molecular Systems Research Unit, University of Oulu, P.O. Box 3000, FI-90014 Oulu, Finland
}

(Received 15 January 2021; revised 26 May 2021; accepted 8 June 2021; published 23 June 2021)

\begin{abstract}
We study the quantum ground-state phases of the one-dimensional disordered Bose-Hubbard model with attractive interactions, realized by a chain of superconducting transmon qubits or cold atoms. We map the phase diagram using perturbation theory and exact diagonalization. Compared to the repulsive Bose-Hubbard model, the quantum ground-state behavior is dramatically different. At strong disorder of the on-site energies, all the bosons localize into the vicinity of a single site, contrary to the Bose glass behavior of the repulsive model. At weak disorder, depending on hopping, the ground state is either superfluid or a $W$ state, which is a multisite and multiparticle entangled superposition of states where all the bosons occupy a single site. We show that the robustness of the $W$ phase against disorder diminishes as the total number of bosons increases.
\end{abstract}

DOI: 10.1103/PhysRevB.103.L220202

\section{INTRODUCTION}

The Bose-Hubbard model is a paradigmatic model of quantum matter and quantum phase transitions, with applications ranging from magnetism to disordered superfluid helium [1-4]. It is canonically characterized by a repulsive bosonboson interaction disfavoring local multi-occupancy, together with boson hopping which models excitation kinetics. When repulsive interaction dominates hopping, the ground state is the Mott insulating phase where a fixed integer number of bosons are located on each lattice site. Otherwise, the ground state is the delocalized superfluid $[5,6]$. In the presence of disorder, a third phase-the Bose glass-emerges between the Mott insulator and the superfluid phases [6-14]. The Bose glass is an insulating phase with finite compressibility caused by disorder localization.

The attractive Bose-Hubbard model has remained much less studied than its repulsive counterpart. At strong disorder, many-body localization emerges [15-17] for highly excited states. The quantum ground-state behavior changes dramatically when switching from repulsive to attractive interaction [18-22]. When attractive interaction dominates hopping, in the absence of disorder, the ground state is the $W$ state, a fascinating self-trapped multiparticle entangled state comprising a cat-state-like superposition of states with all the bosons occupying a single site [23-25]. However, the interplay of disorder and attractive interactions has not been studied before for the quantum ground states.

The attractive Bose-Hubbard model is an important model for arrays of superconducting transmon devices, a leading platform for large-scale quantum science experiments. A transmon is an anharmonic bosonic oscillator with negative anharmonicity [26]. Fabrication disorder [27] has hindered their utilization in large-scale quantum simulators $[28,29]$ of other than disorder physics [15,17,30-32]. The size of experimentally demonstrated transmon arrays has grown rapidly from a few to over 50 sites [30-38]. Thus, an array of coupled transmons realizes the disordered attractive Bose-Hubbard model in a natural manner [16,33]. Furthermore, the attractive Bose-Hubbard model is immediately applicable also for cold atoms in optical lattices, where the interaction can be tuned from repulsive to attractive via the Feshbach resonance [4,39].

In this letter, we use exact diagonalization and perturbation theory to construct the ground-state phase diagram of the onedimensional disordered attractive Bose-Hubbard model, and provide analytical expressions for the states belonging to the $W$ phase, the superfluid phase, and the localized phase. Our main result is that the robustness of the $W$ phase against disorder diminishes exponentially as the total number of bosons is increased. Finally, we propose a possible realization of these phases using transmon chains with experimentally feasible parameters.

We note that phases and phase transitions are, strictly speaking, only defined in the thermodynamic limit, that is, when both the number of lattice sites and the number of particles approach infinity. For the Bose-Hubbard model with attractive interactions, this is ill-defined since the two limits are noncommutative [21] and the ground-state energy is not bounded below. However, seeing that the finite-size behavior of the model resembles that of a system with well-defined phases, the concept of phase is frequently used [21,25,40].

\section{MODEL}

The disordered attractive Bose-Hubbard model with $L$ sites is defined in the basis of the local bosonic annihilation $\hat{a}_{\ell}$, creation $\hat{a}_{\ell}^{\dagger}$, and occupation number $\hat{n}_{\ell}=\hat{a}_{\ell}^{\dagger} \hat{a}_{\ell}$ operators by the Hamiltonian [15,16,33,34]

$$
\frac{\hat{H}}{\hbar}=\sum_{\ell=1}^{L}\left[\omega_{\ell} \hat{n}_{\ell}-\frac{U}{2} \hat{n}_{\ell}\left(\hat{n}_{\ell}-1\right)+J\left(\hat{a}_{\ell+1}^{\dagger} \hat{a}_{\ell}+\hat{a}_{\ell}^{\dagger} \hat{a}_{\ell+1}\right)\right] .
$$

Here, $\omega_{\ell}$ represent random disorder of on-site energies. We draw them from a uniform distribution in the interval $\omega_{\ell} \in$ 


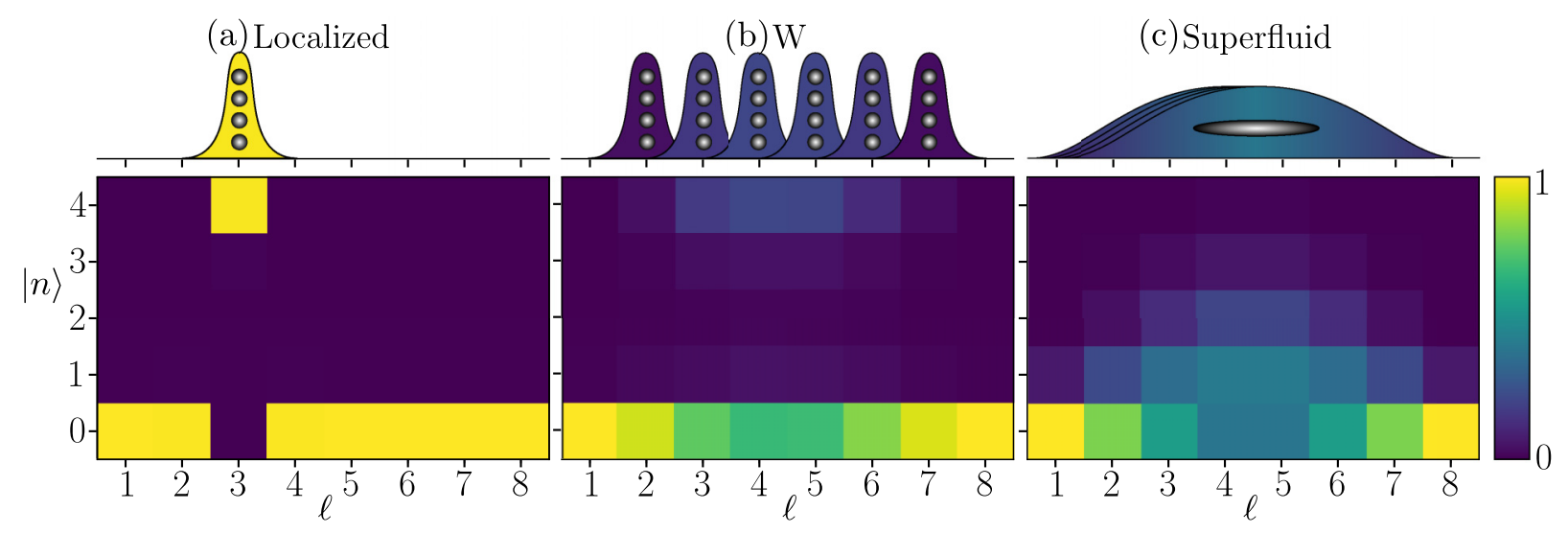

FIG. 1. A schematic illustration and the local occupation density $p_{n \ell}=\left\langle n\left|\hat{\rho}_{\ell}\right| n\right\rangle$ as a function of the transmon site $\ell$ and the local Fock state $|n\rangle$ for (a) the localized state, (b) the $W$ state, and (c) the superfluid state at the scaled hopping frequency $\tau=0.05,0.15$, and 1 , respectively. A single disorder realization at the scaled disorder strength $\delta=0.33 \times 10^{-3}$ was used. The local density matrix $\hat{\rho}_{\ell}$ is calculated by tracing over all the other sites: $\hat{\rho}_{\ell}=\operatorname{Tr}_{\{i \neq \ell\}}(|\psi\rangle\langle\psi|)$, where $|\psi\rangle$ is the ground state.

$[-D, D]$, with $D$ being the strength of the disorder. Since the model conserves the total number of bosons $\hat{N}=\sum_{\ell=1}^{L} \hat{n}_{\ell}$, we consider a fixed $N$ and can thus ignore the mean on-site energy. The strength of the attractive interactions is given by $U$, and $J$ is the hopping frequency. Finally, $\hbar$ is the reduced Planck's constant. The bounded spectrum at fixed $N$ implies that the most excited eigenstate of the Hamiltonian (1) is the quantum ground state of the corresponding repulsive model and vice versa. For the sake of experimental relevance, we focus on open chains with $\hat{a}_{L+1}=0$ in Eq. (1) (see Ref. [41] for the corresponding results for a periodic chain). In what follows, we measure all energies in units of the characteristic energy $\hbar U N(N-1)$, yielding the scaled energy $\varepsilon=E / \hbar U N(N-1)$, hopping frequency $\tau=J / U(N-$ $1)$, disorder strength $\delta=D / U(N-1)$, and on-site energies $\sigma_{\ell}=\omega_{\ell} / U(N-1)$.

\section{LOCALIZED PHASE}

Let us first consider strong attractive interactions and strong disorder $\delta \gg 1 /(N-1) \gg \tau$. The attractive interactions force all the bosons to occupy the site $\ell_{0}$ with the lowest on-site energy $\sigma_{\ell_{0}}$, leading to total energy $\varepsilon_{\text {loc }}^{0}=-1 / 2+\sigma_{\ell_{0}}$. With strong disorder and ignoring hopping, the ground state is a product state of the form

$$
\left|\psi_{\ell_{0}}^{0}\right\rangle=\left|n_{\ell_{0}}=N\right\rangle,
$$

where $\left|n_{\ell_{0}}=N\right\rangle$ denotes the state where $N$ bosons occupy the site $\ell_{0}$ and other sites are empty. We refer to this as the localized state, not to be confused with Anderson localization of the noninteracting situation or the many-body localization of the highly excited states [15-17]. When we take into account the hopping up to first order in nondegenerate perturbation theory, the localized state $\left|\psi_{\ell_{0}}^{0}\right\rangle$ gets a correction of the form

$$
\left|\widetilde{\psi}_{\ell_{0} \pm 1}^{1}\right\rangle=\tau \sqrt{N} \frac{\left|n_{\ell_{0} \pm 1}=1, n_{\ell_{0}}=N-1\right\rangle}{\left(\sigma_{\ell_{0}}-\sigma_{\ell_{0} \pm 1}\right)-1},
$$

that is, a state localized onto the site $\ell_{0}$ will be

$$
\left|\psi_{\ell_{0}}^{1}\right\rangle=\left|\psi_{\ell_{0}}^{0}\right\rangle+\left|\widetilde{\psi}_{\ell_{0}+1}^{1}\right\rangle+\left|\widetilde{\psi}_{\ell_{0}-1}^{1}\right\rangle,
$$

up to a normalization factor [see Fig. 1(a)]. The same form applies to every localized state excepting the ends, where only a single correction is added. After averaging over the disorder, the second-order energy of the localized ground-state phase is

$$
\varepsilon_{\mathrm{loc}}=-\frac{1}{2}-\delta \frac{L-1}{L+1}\left(1-2 \tau^{2}\right)-2 \tau^{2} \frac{L-1}{L} .
$$

When reducing disorder, the ground-state phase changes either into the superfluid phase or into the $W$ phase, depending on hopping.

\section{IV. $W$ PHASE}

Let us next consider weak hopping, $\tau \ll 1$, in the absence of disorder. Then the states $\left|n_{\ell=1, \ldots, L}=N\right\rangle$ with the energy $\varepsilon^{0}=-1 / 2$ are degenerate and coupled by a weak high-order hopping interaction, yielding a quantum ground state which is a superposition of the localized states $\left|\psi_{\ell}^{1}\right\rangle$ of Eq. (4), see Fig. 1(b). This state is called the $W$ state $[25,42,43]$ because of its resemblance with the $W$ state of entangled qubits [44].

To solve the $W$ state analytically, we resort to high-order degenerate perturbation theory [45], detailed in Ref. [41]. First, the sites at the ends of the open chain have only one neighbor each. Thus, the effective hopping energy near the ends is higher than elsewhere, reducing the number of degenerate states by $\ell_{\mathrm{s}}=\lceil N / 2\rceil-1$ counting from both ends of the chain. For simplicity, let us consider even $L$ and odd $N$, such that the number of degenerate states $L_{\mathrm{d}}=L-2 \ell_{\mathrm{s}} \geqslant 2$. Then the degenerate perturbation theory in the $N$ th order shows that the remaining states $\left|n_{\ell}=N\right\rangle$ are each coupled with their neighboring-site counterparts, which gives us the superposition [see Fig. 1(b)]

$$
\left|\psi_{W}^{1}\right\rangle=\sqrt{\frac{2}{L_{d}+1}} \sum_{\ell=1}^{L_{\mathrm{d}}}(-1)^{\ell} \sin \left(\frac{\pi \ell}{L_{d}+1}\right)\left|\psi_{\ell+\ell_{\mathrm{s}}}^{1}\right\rangle .
$$

The existence of the $W$ state requires a nonzero hopping frequency, which is why we use the first-order perturbed states of Eq. (4) in the superposition. The shape of the $W$ state depends strongly on the total density $N / L$ of the bosonsthe higher the density, the more the bosons bunch toward 
the middle of the chain, forming a self-trapping state [22]. For even $L$, the limit is an equal superposition of the two middlemost localized states of Eq. (4). For odd $L$, the limit is just the middlemost localized state. The mean second-order energy of the $W$ state is

$$
\varepsilon_{W}=-\frac{1}{2}-2 \tau^{2},
$$

with no disorder contribution after averaging over different realizations.

When introducing disorder, we can probe the weak interaction between the degenerate states formed through high-order bosonic hopping. The magnitude of the $N$ th order hopping interaction between the state $\left|n_{\ell}=N\right\rangle$ and the states $\left|n_{\ell \pm 1}=N\right\rangle$ is $[\alpha(N) \tau]^{N}$, where $\alpha(N)=\left[(N-1)^{N-1} /(N-1) !\right]^{1 / N}$ is a coefficient dependent on the boson number. At large $N$, the value of $\alpha(N)$ approaches Euler's number $e$. The magnitude of disorder needs to be of the same order or stronger than this effective hopping energy to cause the $W$ state to disintegrate into a localized state. To sum up, at weak disorder and hopping, the $W$ phase can exist only when $\delta \lesssim[\alpha(N) \tau]^{N}$, showing that the robustness of the $W$ phase against disorder diminishes exponentially with increasing total boson number.

\section{SUPERFLUID PHASE}

When hopping dominates interactions, $\tau \gg 1$, the bosons can move from site to site largely unhindered by each other, that is, the ground state is a superfluid. It is easier here to work in the reciprocal space, i.e., in the eigenbasis of the hopping term of the Hamiltonian, accessible via the transformation

$$
\hat{c}_{k}=\sqrt{\frac{2}{L+1}} \sum_{\ell=1}^{L} \sin \left(\frac{\pi \ell k}{L+1}\right) \hat{a}_{\ell}
$$

in the open chain. Analogously to the spatially localized states of Eq. (2), we may form localized states in the reciprocal space

$$
\left|\psi_{k}^{0}\right\rangle=\left|\eta_{k}=N\right\rangle
$$

where $\left|\eta_{k}=N\right\rangle$ denotes the state where $N$ bosons occupy the $k$ th reciprocal mode and other modes are empty. In the limit of vanishing interactions and disorder, the superfluid ground state $\left|\psi_{\mathrm{SF}}^{0}\right\rangle=\left|\psi_{k=L}^{0}\right\rangle$ is completely localized to the lowest-energy mode of the reciprocal space, $k=L$, with the energy $\varepsilon_{\mathrm{SF}}^{0}=-2 \tau \cos [\pi /(L+1)]$. Although the excitations created via $\hat{c}_{k}^{\dagger}$ are localized in the reciprocal space, they are delocalized in the position space, see Fig. 1(c) and Ref. [41] for visualizations. Both the interaction term and the disorder term have a weakly delocalizing effect in the reciprocal space, similar to the effect of the hopping term on the spatially localized state. The ground state can be calculated by first transforming these into the reciprocal basis and considering their effects perturbatively [41]. The second-order disorderaveraged energy is given by

$$
\varepsilon_{\mathrm{SF}}=-2 \tau \cos \left(\frac{\pi}{L+1}\right)-\frac{3}{4(L+1)}-\frac{a}{\tau}\left(\delta^{2}+b\right),
$$

where $a$ and $b$ are coefficients dependent on $N$ and $L$ (at $N=4$ and $L=8, a \approx 0.23$ and $b \approx 0.05$, see Ref. [41]). Notice that this condensate of bosons can indeed be called a superfluid since it is able to support metastable persistent currents in a periodic chain $[46,47]$.

\section{PHASE DIAGRAM}

Analytical results indicate that we could expect groundstate phase transitions at parameters where the energies of any two phases [Eqs. (5), (7), and (10)] are close. To study this in detail, we calculate the quantum ground state of the Hamiltonian (1) by numerical exact diagonalization. To identify all three phases in numerical calculations, we need at least two indicator quantities. We can use the fact that the states are characterized by different types of localization: the localized and superfluid phases are localized in spatial and reciprocal bases, respectively, and the $W$ state is a superposition of spatially localized states. One quantity measuring the degree of localization is the inverse participation ratio

$$
\mathcal{P}_{\mathrm{s} / \mathrm{r}}=\frac{1}{L-1}\left(\frac{N^{2}}{\sum_{m=1}^{L}\left|\left\langle\psi\left|\hat{n}_{m}^{\mathrm{s} / \mathrm{r}}\right| \psi\right\rangle\right|^{2}}-1\right),
$$

which we can calculate in both the position space $\left(\mathcal{P}_{\mathrm{s}}\right)$ and in the reciprocal space $\left(\mathcal{P}_{\mathrm{r}}\right)$ separately. Here, $\hat{n}_{m}^{\mathrm{s}}=\hat{a}_{m}^{\dagger} \hat{a}_{m}$ and $\hat{n}_{m}^{\mathrm{r}}=\hat{c}_{m}^{\dagger} \hat{c}_{m}$ are the number operators in the corresponding spaces. The inverse participation ratio yields zero if the state is localized and one if the state is completely delocalized. We can use $\mathcal{P}_{\mathrm{s}}$ to distinguish the spatially localized states from the superfluid states and the $W$ states. Conversely, we can use $\mathcal{P}_{\mathrm{r}}$ to distinguish the superfluid states from the $W$ states and the localized states.

Keeping in mind the theoretical limitations of increasing the total boson number $N$ mentioned in the Introduction, we are nevertheless interested in the phase boundaries of the three phases as the system size in $N$ is increased, which physically means approaching the semiclassical limit. For this purpose, we define the critical scaled hopping frequency [48]

$$
\tau_{\mathrm{c}}^{\mathrm{s} / \mathrm{r}}=\underset{\tau}{\operatorname{argmax}}\left|\frac{\partial \mathcal{P}_{\mathrm{s} / \mathrm{r}}}{\partial \tau}\right|
$$

to locate the transition point from localized to delocalized state both in the position space $\left(\tau_{\mathrm{c}}^{\mathrm{s}}\right)$ and in the reciprocal space $\left(\tau_{\mathrm{c}}^{\mathrm{r}}\right)$.

In Fig. 2(a), we show the inverse participation ratios $\mathcal{P}_{\mathrm{s} / \mathrm{r}}$ for the ground states of the Hamiltonian (1) as a function of the scaled hopping frequency $\tau$ at weak disorder. The critical scaled hopping frequencies $\tau_{\mathrm{c}}^{\mathrm{s} / \mathrm{r}}$ indicate that the system localizes in the position space (solid) when $\tau<\tau_{\mathrm{c}}^{\mathrm{s}} \approx 0.11$ and in the reciprocal space (dashed) when $\tau>\tau_{\mathrm{c}}^{\mathrm{r}} \approx 0.17$. In the region between, we identify the $W$ state which is delocalized spatially but not yet localized in the reciprocal space. Note that $\mathcal{P}_{\mathrm{r}}$ attains its maximum at a nonzero $\tau$, within the $W$ phase. This stems from the fact that, due to the shape of the transformation (8), a superposition of spatially localized states can be more uniformly distributed in the reciprocal space than a single localized state. However, as the number of bosons $N$ grows, the $W$ state gets more and more localized towards the middle of the chain, and thus the local maximum of $\mathcal{P}_{\mathrm{r}}$ at $\tau>0$ disappears. At moderate disorder, depicted in Fig. 2(b), increasing hopping simultaneously delocalizes the system in 

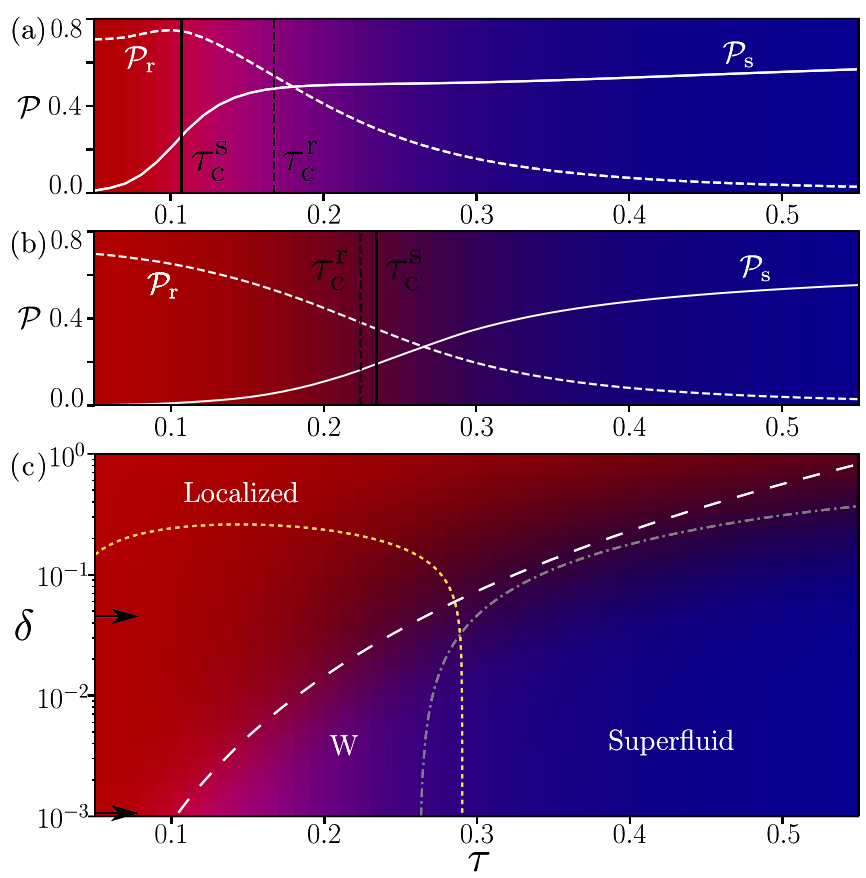

FIG. 2. (a), (b) In white, the inverse participation ratios $\mathcal{P}_{\mathrm{s}}$ (solid) and $\mathcal{P}_{\mathrm{r}}$ (dashed), defined in Eq. (11), of the quantum ground state of the disordered attractive Bose-Hubbard model (1) as a function of the scaled hopping frequency $\tau$ at two values of the scaled disorder strength, (a) $\delta=0.001$ and (b) $\delta=0.036$. In black, the corresponding critical scaled hopping frequencies $\tau_{\mathrm{c}}^{\mathrm{s} / \mathrm{r}}$ defined in Eq. (12). (c) The two inverse participation ratios $\mathcal{P}_{\mathrm{s} / \mathrm{r}}$, represented as a single colormap, as a function of the scaled hopping frequency $\tau$ and the disorder strength $\delta$. The amount of blue (red) indicates the value of $\mathcal{P}_{\mathrm{s}}\left(\mathcal{P}_{\mathrm{r}}\right)$ : The bluer (redder) the pixel, the more delocalized the ground state is spatially (in the reciprocal space). When both of the inverse participation ratios are nonzero, the color of the corresponding pixel is purple, indicating the $W$ phase. The overlaid lines indicate the phase boundaries estimated from the analytical results: localizedto- $W$ at $\delta=2(\alpha \tau)^{N}$ (white dashed line), superfluid-to-localized at $\varepsilon_{\mathrm{SF}}=\varepsilon_{\text {loc }}$ (gray dash-dotted line), and $W$-to-superfluid at $\varepsilon_{\mathrm{W}}=\varepsilon_{\mathrm{SF}}$ (yellow dotted line). The arrows indicate the locations of the horizontal cutoffs shown in (a) and (b). The ground states were numerically computed for an open chain of length $L=8$ with the total number of bosons $N=4$, and averaged over 1000 disorder realizations. See Ref. [41] for the results for corresponding periodic chains. Note that $\tau \geqslant 0.05$ in this and all the following figures.

the position space and localizes it in the reciprocal space, with a crossover at $\tau \approx 0.23$, indicating absence of the $W$ state.

To explore the ground-state phases in the full parameter space, we computed the inverse participation ratios $\mathcal{P}_{\mathrm{s} / \mathrm{r}}$ as a function of both the scaled hopping frequency $\tau$ and the scaled disorder strength $\delta$. These are represented in Fig. 2(c) as a colormap, where the amount of blue (red) color in each pixel indicates the value of $\mathcal{P}_{\mathrm{s}}\left(\mathcal{P}_{\mathrm{r}}\right)$. In the $W$ phase, both indicators are nonzero, shown with purple color. Notice that with $N=4$ and $L=8$, the $W$ phase exists only at $\delta \lesssim 0.03$. At stronger disorder, there is a direct transition from the localized phase into the superfluid phase. The overlaid lines of Fig. 2(c) indicate the phase boundaries predicted by equating the analytical ground-state energies.
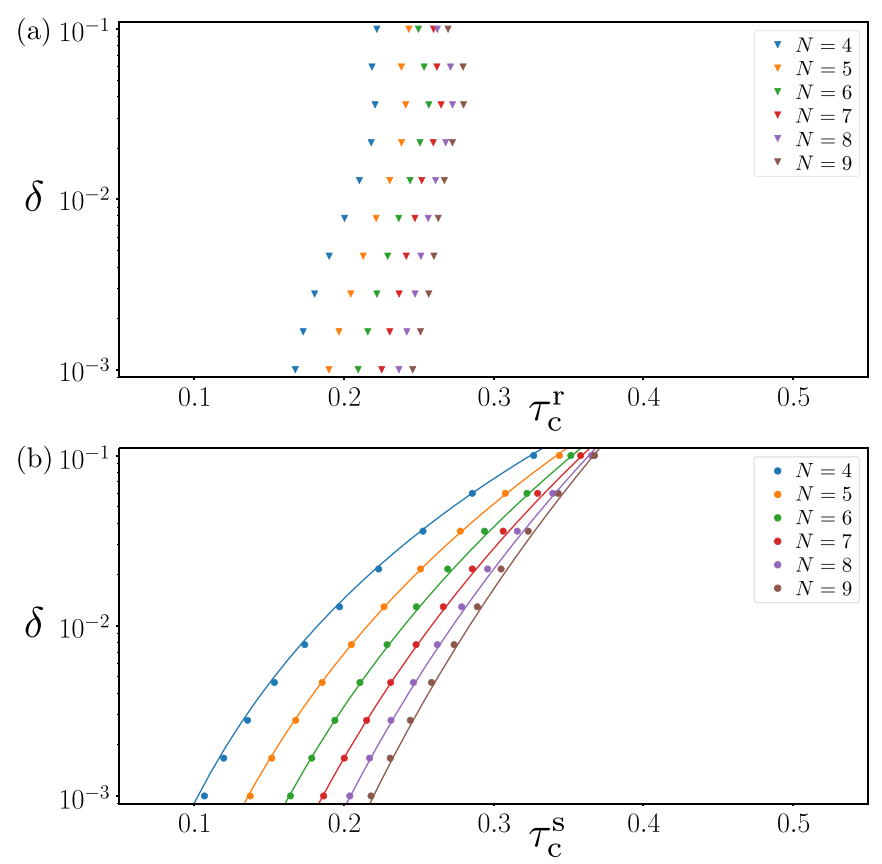

FIG. 3. The critical scaled hopping frequencies (a) $\tau_{\mathrm{c}}^{\mathrm{r}}$ (colored filled triangles) and (b) $\tau_{\mathrm{c}}^{\mathrm{s}}$ (colored filled circles), defined in Eq. (12), as a function of the total boson number $N=4, \ldots, 9$ and the scaled disorder strength $\delta$. The chain length is $L=8$ and the results are averaged over 2000 disorder realizations. The analytically predicted phase boundary between the localized phase and the $W$ phase is shown as colored lines at $\delta=2\left[\alpha(N) \tau_{\mathrm{c}}^{\mathrm{s}}\right]^{N}$ in (b).

At strong disorder, the localized phase changes into the superfluid phase near the curve $\varepsilon_{\text {loc }}=\varepsilon_{\mathrm{SF}}$ (dash-dotted line). At weak disorder, the superfluid phase changes into the $W$ phase in the proximity of the curve $\varepsilon_{\mathrm{SF}}=\varepsilon_{\mathrm{W}}$ (dotted line), while the $W$ phase disintegrates into the localized phase at $\delta=2[\alpha(N) \tau]^{N}$ (dashed line). Here, the numerical coefficient 2 in the localized-to- $W$ boundary was obtained by fitting the analytically predicted expression (see Ref. [41]) $\delta=(3 A / 2)[\alpha(N) \tau]^{N}$, with $A$ a constant of the order of unity, to the numerical data.

Figures 3(a) and 3(b) show the critical hopping frequencies $\tau_{\mathrm{c}}^{\mathrm{r}}$ and $\tau_{\mathrm{c}}^{\mathrm{s}}$, respectively, as a function of the disorder strength $\delta$ for varying total boson number $N$. In Fig. 3(a), the transition between the $W$ phase and the superfluid phase shifts towards larger hopping frequency as $N$ is increased. We can explain this qualitatively through the fact that the coupling between the reciprocal modes by the interaction term of the Hamiltonian (1) increases with $N$, contained in the coefficients $a$ and $b$ of Eq. (10). In Fig. 3(b), the data points indicate the transition between the localized and the $W$ phase. Here, we observe clearly that the $W$ phase exists only at $\delta \lesssim 2[\alpha(N) \tau]^{N}$ (solid colored lines), confirming our analytical prediction.

\section{REALIZATION WITH TRANSMON ARRAYS}

With transmon arrays, the hopping term is realized by the capacitive coupling between the transmons [26,29], resulting in the value of $J / 2 \pi$ ranging between $10 \mathrm{MHz}$ and $100 \mathrm{MHz}$. The interaction term originates from an approximation of 
the cosinusoidal potential of the Josephson junctions of the transmons [26], with typical values of $U / 2 \pi$ between $200 \mathrm{MHz}$ and $300 \mathrm{MHz}$. The disorder $\omega_{\ell}$ models the small unintentional variations in manufactured devices, but it can also be artificially magnified or reduced by applying magnetic flux through the loop formed by the two parallel Josephson junctions of the transmons $[15,16,26,33,34,36]$, yielding $D / 2 \pi$ in the range from $100 \mathrm{kHz}$ to $2 \mathrm{GHz}$. The experimentally realistic range of parameters belongs roughly to the interval $J / U \in$ $[0.03,0.5]$ and $D / U \in\left[10^{-4}, 10^{2}\right]$, allowing the realization of all the ground-state phases of the disordered attractive Bose-Hubbard model. For modern transmons, the rate $\Gamma_{1}$ of losing bosons (photons) is low $[32,34,37]$, with $\Gamma_{1} / 2 \pi$ in the range of a few $\mathrm{kHz}$. This should be contrasted with the effective hopping frequencies in the system. For example, in the $W$ phase, the ratio between the effective hopping frequency $\widetilde{J}=U N(N-1)[\alpha(N) \tau]^{N}$ and the many-body dissipation rate is $\widetilde{J} /\left(N \Gamma_{1}\right) \gtrsim 50$ with the parameters of Ref. [37], indicating an ample window of coherent dynamics to form and detect the $W$ phase before a disruptive photon loss event.

The Hamiltonian (1) becomes increasingly worse a model of a transmon array as the number of bosons on a single site increases, since the interaction term $-U \hat{n}(\hat{n}-1) / 2$ is just the lowest anharmonicity term of the cosine potential of the Josephson junction [26]. However, a more fundamental limitation is that the cosine potential also implies that a transmon has only a finite number of discrete bound states [49]. This number for modern transmon parameters is $\omega_{0} /(\sqrt{8} U) \approx 10$, with $\omega_{0}$ the mean on-site energy. Adding more bosons will change the transmon spectrum and couplings, rendering the Bose-Hubbard model insufficient. This warrants our choice of $N<10$.

To detect and distinguish the state, the population of every transmon or a subset of them can be measured with high accuracy by coupling them individually to dispersive readout resonators [50], as demonstrated recently in many-body settings [15,34]. This way, one can measure the local occupation density that uniquely identifies each phase as shown in Fig. 1. By utilizing the sophisticated driving protocols, one can realize cooling and stabilization schemes that can be used to achieve the quantum ground states at fixed boson numbers, as was experimentally demonstrated in the seminal works $[33,34]$.

\section{CONCLUSION}

In this work, we concentrated on the static ground-state properties of the attractive Bose-Hubbard model with weakto-moderate energy disorder. We mapped the ground-state phase diagram and focused on the $W$ phase, which can be experimentally realized with transmon arrays only within stringent bounds for the disorder and coupling strengths. Seeing that decoherence and dissipation are common issues in superconducting quantum devices, and that the loss of bosons can change the nature of the ground state, even a system starting from the ground state for a given number of bosons $N$ may experience quite interesting dynamics. Specifically, even if the bare system parameters $J$ and $U$ are not changed, boson losses due to dissipation effectively act as quenches in the scaled hopping frequency $\tau=J / U(N-1)$ and disorder strength $\delta=D / U(N-1)$. This allows for the exploration of the parameter space $(\tau, \delta)$, although only in discrete steps. A more detailed discussion of the dynamics due to dissipation and quenches in the parameters of the model, including a potential realization of dynamical quantum phase transition [51,52], is an interesting subject of future work. Another intriguing question is the structure of the ground-state phase diagram in two and higher dimensions, relevant even for current transmon arrays [36].

\section{ACKNOWLEDGMENTS}

The authors would like to thank Steven Girvin and Tuure Orell for useful discussions on this and other closely related topics. This research work was financially supported by the Alfred Kordelin foundation, the Emil Aaltonen foundation, the Kvantum Institute of the University of Oulu, and the Academy of Finland under Grants No. 316619 and No. 320086. The numerics are implemented in JULIA [53] using the KRYLOvKIT and OPTIM [54] packages.
[1] S. M. Girvin and K. Yang, Modern Condensed Matter Physics (Cambridge University Press, Cambridge, England, 2019).

[2] M. Greiner, O. Mandel, T. Esslinger, T. W. Hänsch, and I. Bloch, Quantum phase transition from a superfluid to a Mott insulator in a gas of ultracold atoms, Nature 415, 39 (2002).

[3] T. Stöferle, H. Moritz, C. Schori, M. Köhl, and T. Esslinger, Transition from a Strongly Interacting 1D Superfluid to a Mott Insulator, Phys. Rev. Lett. 92, 130403 (2004).

[4] I. Bloch, J. Dalibard, and W. Zwerger, Many-body physics with ultracold gases, Rev. Mod. Phys. 80, 885 (2008).

[5] T. D. Kühner, S. R. White, and H. Monien, One-dimensional Bose-Hubbard model with nearest-neighbor interaction, Phys. Rev. B 61, 12474 (2000).

[6] M. A. Cazalilla, R. Citro, T. Giamarchi, E. Orignac, and M. Rigol, One dimensional bosons: From condensed matter systems to ultracold gases, Rev. Mod. Phys. 83, 1405 (2011).
[7] T. Giamarchi and H. J. Schulz, Anderson localization and interactions in one-dimensional metals, Phys. Rev. B 37, 325 (1988).

[8] M. P. A. Fisher, P. B. Weichman, G. Grinstein, and D. S. Fisher, Boson localization and the superfluid-insulator transition, Phys. Rev. B 40, 546 (1989).

[9] D. B. Haviland, Y. Liu, and A. M. Goldman, Onset of Superconductivity in the Two-Dimensional Limit, Phys. Rev. Lett. 62, 2180 (1989).

[10] A. F. Hebard and M. A. Paalanen, Magnetic-Field-Tuned Superconductor-Insulator Transition in Two-Dimensional Films, Phys. Rev. Lett. 65, 927 (1990).

[11] S. Rapsch, U. Schollwoeck, and W. Zwerger, Density matrix renormalization group for disordered bosons in one dimension, Europhys. Lett. 46, 559 (1999).

[12] L. Fallani, J. E. Lye, V. Guarrera, C. Fort, and M. Inguscio, Ultracold Atoms in a Disordered Crystal of 
Light: Towards a Bose Glass, Phys. Rev. Lett. 98, 130404 (2007).

[13] R. Yu, L. Yin, N. S. Sullivan, J. S. Xia, C. Huan, A. PaduanFilho, N. F. Oliveira, Jr., S. Haas, A. Steppke, C. F. Miclea, F. Weickert, R. Movshovich, E.-D. Mun, B. L. Scott, V. S. Zapf, and T. Roscilde, Bose glass and Mott glass of quasiparticles in a doped quantum magnet, Nature 489, 379 (2012).

[14] C. Meldgin, U. Ray, P. Russ, D. Chen, D. M. Ceperley, and B. DeMarco, Probing the Bose glass-superfluid transition using quantum quenches of disorder, Nat. Phys. 12, 646 (2016).

[15] P. Roushan, C. Neill, J. Tangpanitanon, V. M. Bastidas, A. Megrant, R. Barends, Y. Chen, Z. Chen, B. Chiaro, A. Dunsworth, A. Fowler, B. Foxen, M. Giustina, E. Jeffrey, J. Kelly, E. Lucero, J. Mutus, M. Neeley, C. Quintana, D. Sank, A. Vainsencher, J. Wenner, T. White, H. Neven, D. G. Angelakis, and J. Martinis, Spectroscopic signatures of localization with interacting photons in superconducting qubits, Science 358, 1175 (2017).

[16] T. Orell, A. A. Michailidis, M. Serbyn, and M. Silveri, Probing the many-body localization phase transition with superconducting circuits, Phys. Rev. B 100, 134504 (2019).

[17] B. Chiaro, C. Neill, A. Bohrdt, M. Filippone, F. Arute, K. Arya, R. Babbush, D. Bacon, J. Bardin, R. Barends, S. Boixo, D. Buell, B. Burkett, Y. Chen, Z. Chen, R. Collins, A. Dunsworth, E. Farhi, A. Fowler, B. Foxen, C. Gidney, M. Giustina, M. Harrigan, T. Huang, S. Isakov, E. Jeffrey, Z. Jiang, D. Kafri, K. Kechedzhi, J. Kelly, P. Klimov, A. Korotkov, F. Kostritsa, D. Landhuis, E. Lucero, J. McClean, X. Mi, A. Megrant, M. Mohseni, J. Mutus, M. McEwen, O. Naaman, M. Neeley, M. Niu, A. Petukhov, C. Quintana, N. Rubin, D. Sank, K. Satzinger, A. Vainsencher, T. White, Z. Yao, P. Yeh, A. Zalcman, V. Smelyanskiy, H. Neven, S. Gopalakrishnan, D. Abanin, M. Knap, J. Martinis, and P. Roushan, Direct measurement of non-local interactions in the many-body localized phase, arXiv:1910.06024.

[18] J. Dorignac, J. C. Eilbeck, M. Salerno, and A. C. Scott, Quantum Signatures of Breather-Breather Interactions, Phys. Rev. Lett. 93, 025504 (2004).

[19] M. W. Jack and M. Yamashita, Bose-Hubbard model with attractive interactions, Phys. Rev. A 71, 023610 (2005).

[20] P. Buonsante, V. Penna, and A. Vezzani, Attractive ultracold bosons in a necklace optical lattice, Phys. Rev. A 72, 043620 (2005).

[21] N. Oelkers and J. Links, Ground-state properties of the attractive one-dimensional Bose-Hubbard model, Phys. Rev. B 75, 115119 (2007)

[22] P. Buonsante, V. Penna, and A. Vezzani, Quantum signatures of the self-trapping transition in attractive lattice bosons, Phys. Rev. A 82, 043615 (2010).

[23] L. Bernstein, J. C. Eilbeck, and A. C. Scott, The quantum theory of local modes in a coupled system of nonlinear oscillators, Nonlinearity 3, 293 (1990).

[24] O. S. Sørensen, S. Gammelmark, and K. Mølmer, Relative and center-of-mass motion in the attractive Bose-Hubbard model, Phys. Rev A 85, 043617 (2012).

[25] A. A. Gangat, I. P. McCulloch, and G. J. Milburn, Deterministic Many-Resonator W Entanglement of Nearly Arbitrary Microwave States Via Attractive Bose-Hubbard Simulation, Phys. Rev. X 3, 031009 (2013).
[26] J. Koch, T. M. Yu, J. Gambetta, A. A. Houck, D. I. Schuster, J. Majer, A. Blais, M. H. Devoret, S. M. Girvin, and R. J. Schoelkopf, Charge-insensitive qubit design derived from the Cooper pair box, Phys. Rev. A 76, 042319 (2007).

[27] D. L. Underwood, W. E. Shanks, J. Koch, and A. A. Houck, Low-disorder microwave cavity lattices for quantum simulation with photons, Phys. Rev. A 86, 023837 (2012).

[28] A. A. Houck, H. E. Türeci, and J. Koch, On-chip quantum simulation with superconducting circuits, Nat. Phys. 8, 292 (2012).

[29] M. Dalmonte, S. I. Mirzaei, P. R. Muppalla, D. Marcos, P. Zoller, and G. Kirchmair, Realizing dipolar spin models with arrays of superconducting qubits, Phys. Rev. B 92, 174507 (2015).

[30] K. Xu, J.-J. Chen, Y. Zeng, Y.-R. Zhang, C. Song, W. Liu, Q. Guo, P. Zhang, D. Xu, H. Deng, K. Huang, H. Wang, X. Zhu, D. Zheng, and H. Fan, Emulating Many-Body Localization with a Superconducting Quantum Processor, Phys. Rev. Lett. 120, 050507 (2018).

[31] C. Zha, V. M. Bastidas, M. Gong, Y. Wu, H. Rong, R. Yang, Y. Ye, S. Li, Q. Zhu, S. Wang, Y. Zhao, F. Liang, J. Lin, Y. Xu, C.-Z. Peng, J. Schmiedmayer, K. Nemoto, H. Deng, W. J. Munro, X. Zhu, and J.-W. Pan, Ergodic-Localized Junctions in a Periodically Driven Spin Chain, Phys. Rev. Lett. 125, 170503 (2020).

[32] M. Gong, G. D. D. M. Neto, C. Zha, Y. Wu, H. Rong, Y. Ye, S. Li, Q. Zhu, S. Wang, Y. Zhao, F. Liang, J. Lin, Y. Xu, C.-Z. Peng, H. Deng, A. Bayat, X. Zhu, and J.-W. Pan, Experimental characterization of quantum many-body localization transition, arXiv:2012.11521.

[33] S. Hacohen-Gourgy, V. V. Ramasesh, C. De Grandi, I. Siddiqi, and S. M. Girvin, Cooling and Autonomous Feedback in a Bose-Hubbard Chain with Attractive Interactions, Phys. Rev. Lett. 115, 240501 (2015).

[34] R. Ma, B. Saxberg, C. Owens, N. Leung, Y. Lu, J. Simon, and D. I. Schuster, A dissipatively stabilized Mott insulator of photons, Nature 566, 51 (2019).

[35] M. Reagor, C. B. Osborn, N. Tezak, A. Staley, G. Prawiroatmodjo, M. Scheer, N. Alidoust, E. A. Sete, N. Didier, M. P. d. Silva, E. Acala, J. Angeles, A. Bestwick, M. Block, B. Bloom, A. Bradley, C. Bui, S. Caldwell, L. Capelluto, R. Chilcott, J. Cordova, G. Crossman, M. Curtis, S. Deshpande, T. E. Bouayadi, D. Girshovich, S. Hong, A. Hudson, P. Karalekas, K. Kuang, M. Lenihan, R. Manenti, T. Manning, J. Marshall, Y. Mohan, W. O'Brien, J. Otterbach, A. Papageorge, J.-P. Paquette, M. Pelstring, A. Polloreno, V. Rawat, C. A. Ryan, R. Renzas, N. Rubin, D. Russel, M. Rust, D. Scarabelli, M. Selvanayagam, R. Sinclair, R. Smith, M. Suska, T.-W. To, M. Vahidpour, N. Vodrahalli, T. Whyland, K. Yadav, W. Zeng, and C. T. Rigetti, Demonstration of universal parametric entangling gates on a multi-qubit lattice, Sci. Adv. 4, eaao3603 (2018).

[36] F. Arute, K. Arya, R. Babbush, D. Bacon, J. C. Bardin, R. Barends, R. Biswas, S. Boixo, F. G. S. L. Brandao, D. A. Buell, B. Burkett, Y. Chen, Z. Chen, B. Chiaro, R. Collins, W. Courtney, A. Dunsworth, E. Farhi, B. Foxen, A. Fowler, C. Gidney, M. Giustina, R. Graff, K. Guerin, S. Habegger, M. P. Harrigan, M. J. Hartmann, A. Ho, M. Hoffmann, T. Huang, T. S. Humble, S. V. Isakov, E. Jeffrey, Z. Jiang, D. Kafri, K. Kechedzhi, J. Kelly, P. V. Klimov, S. Knysh, A. Korotkov, F. Kostritsa, D. Landhuis, M. Lindmark, E. Lucero, D. Lyakh, S. 
Mandrà, J. R. McClean, M. McEwen, A. Megrant, X. Mi, K. Michielsen, M. Mohseni, J. Mutus, O. Naaman, M. Neeley, C. Neill, M. Y. Niu, E. Ostby, A. Petukhov, J. C. Platt, C. Quintana, E. G. Rieffel, P. Roushan, N. C. Rubin, D. Sank, K. J. Satzinger, V. Smelyanskiy, K. J. Sung, M. D. Trevithick, A. Vainsencher, B. Villalonga, T. White, Z. J. Yao, P. Yeh, A. Zalcman, H. Neven, and J. M. Martinis, Quantum supremacy using a programmable superconducting processor, Nature $\mathbf{5 7 4}$, 505 (2019).

[37] D. L. Campbell, Y.-P. Shim, B. Kannan, R. Winik, D. K. Kim, A. Melville, B. M. Niedzielski, J. L. Yoder, C. Tahan, S. Gustavsson, and W. D. Oliver, Universal Nonadiabatic Control of Small-Gap Superconducting Qubits, Phys. Rev. X 10, 041051 (2020).

[38] Q. Guo, C. Cheng, H. Li, S. Xu, P. Zhang, Z. Wang, C. Song, W. Liu, W. Ren, H. Dong, R. Mondaini, and H. Wang, Stark many-body localization on a superconducting quantum processor, arXiv:2011.13895.

[39] M. Theis, G. Thalhammer, K. Winkler, M. Hellwig, G. Ruff, R. Grimm, and J. H. Denschlag, Tuning the Scattering Length with an Optically Induced Feshbach Resonance, Phys. Rev. Lett. 93, 123001 (2004).

[40] X.-L. Deng, D. Porras, and J. I. Cirac, Quantum phases of interacting phonons in ion traps, Phys. Rev. A 77, 033403 (2008).

[41] See Supplemental Material at http://link.aps.org/supplemental/ 10.1103/PhysRevB.103.L220202 for more information.

[42] L. Heaney, A. Cabello, M. F. Santos, and V. Vedral, Extreme nonlocality with one photon, New J. Phys. 13, 053054 (2011).

[43] Z. Wang and D. Markham, Nonlocality and entanglement for symmetric states, Phys. Rev. A 87, 012104 (2013).
[44] W. Dür, G. Vidal, and J. I. Cirac, Three qubits can be entangled in two inequivalent ways, Phys. Rev. A 62, 062314 (2000).

[45] J. J. Sakurai and J. Napolitano, Modern Quantum Mechanics 2nd ed. (Cambridge University Press, Cambridge, England, 2017).

[46] G. Arwas, A. Vardi, and D. Cohen, Superfluidity and chaos in low dimensional circuits, Sci. Rep. 5, 13433 (2015).

[47] G. Arwas and D. Cohen, Superfluidity in Bose-Hubbard circuits, Phys. Rev. B 95, 054505 (2017).

[48] P. Buonsante and A. Vezzani, Ground-State Fidelity and Bipartite Entanglement in the Bose-Hubbard Model, Phys. Rev. Lett. 98, 110601 (2007).

[49] I. Pietikäinen, J. Tuorila, D. S. Golubev, and G. S. Paraoanu, Photon blockade and the quantum-to-classical transition in the driven-dissipative Josephson pendulum coupled to a resonator, Phys. Rev. A 99, 063828 (2019).

[50] P. Krantz, M. Kjaergaard, F. Yan, T. P. Orlando, S. Gustavsson, and W. D. Oliver, A quantum engineer's guide to superconducting qubits, App. Phys. Rev. 6, 021318 (2019).

[51] M. Heyl, Dynamical quantum phase transitions: A review, Rep. Prog. Phys. 81, 054001 (2018).

[52] M. Lacki and M. Heyl, Dynamical quantum phase transitions in collapse and revival oscillations of a quenched superfluid, Phys. Rev. B 99, 121107(R) (2019).

[53] J. Bezanson, A. Edelman, S. Karpinski, and V. B. Shah, Julia: A fresh approach to numerical computing, SIAM Rev. 59, 65 (2017).

[54] P. K. Mogensen and A. N. Riseth, Optim: A mathematical optimization package for Julia, J. Open Source Softw. 3, 615 (2018). 